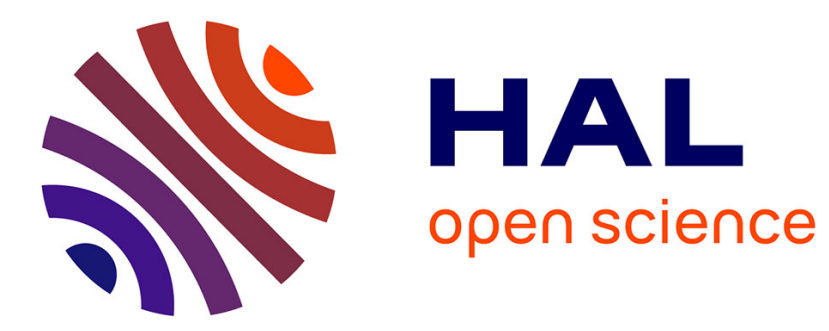

\title{
Investigation of Phason Statics and Dynamics
}

Hans-Rainer Trebin, Ulrich Koschella, Masahiro Umezaki, T. Odagaki

\section{To cite this version:}

Hans-Rainer Trebin, Ulrich Koschella, Masahiro Umezaki, T. Odagaki. Investigation of Phason Statics and Dynamics. Philosophical Magazine, 2006, 86 (06-08), pp.1021-1028. 10.1080/14786430500256409 . hal-00513578

\section{HAL Id: hal-00513578 \\ https://hal.science/hal-00513578}

Submitted on 1 Sep 2010

HAL is a multi-disciplinary open access archive for the deposit and dissemination of scientific research documents, whether they are published or not. The documents may come from teaching and research institutions in France or abroad, or from public or private research centers.
L'archive ouverte pluridisciplinaire HAL, est destinée au dépôt et à la diffusion de documents scientifiques de niveau recherche, publiés ou non, émanant des établissements d'enseignement et de recherche français ou étrangers, des laboratoires publics ou privés. 


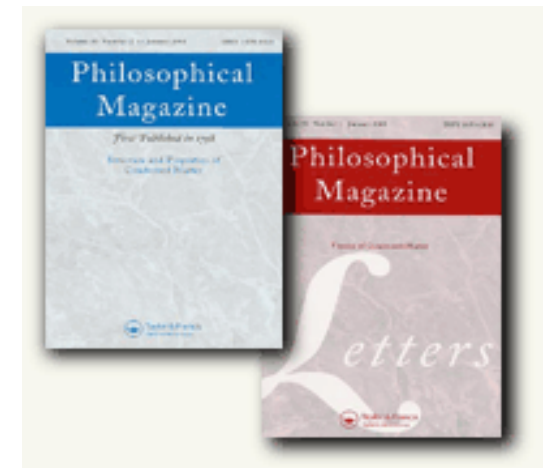

\section{Investigation of Phason Statics and Dynamics}

\begin{tabular}{|c|c|}
\hline Journal: & Philosophical Magazine \& Philosophical Magazine Letters \\
\hline Manuscript ID: & TPHM-05-May-0239 \\
\hline Journal Selection: & Philosophical Magazine \\
\hline $\begin{array}{r}\text { Date Submitted by the } \\
\text { Author: }\end{array}$ & 15-May-2005 \\
\hline Complete List of Authors: & $\begin{array}{l}\text { Trebin, Hans-Rainer; Universität Stuttgart, Institut für Theoretische } \\
\text { und Angewandte Physik } \\
\text { Koschella, Ulrich; Universität Stuttgart, Institut für Theoretische } \\
\text { und Angewandte Physik } \\
\text { Umezaki, Masahiro; Kyushu University, Department of Physics } \\
\text { Odagaki, T.; Kyushu University, Department of Physics }\end{array}$ \\
\hline Keywords: & atomic dynamics, quasicrystals, computer modelling \\
\hline Keywords (user supplied): & phason elasticity \\
\hline
\end{tabular}

\section{(5) ScholaroNE Manuscript Central}




\title{
Investigation of Phason Statics and Dynamics
}

\author{
Hans-Rainer Trebin ${ }^{1}$, Ulrich Koschella ${ }^{1}$, \\ Masahiro Umezaki ${ }^{2}$, Takashi Odagaki ${ }^{2}$ \\ ${ }^{1}$ Institut für Theoretische und Angewandte Physik \\ Universität Stuttgart, Pfaffenwaldring 57, D-70550 Stuttgart, Germany \\ ${ }^{2}$ Department of Physics, Faculty of Science \\ Kyushu University, Fukuoka 812, Japan
}

May 15, 2005

\begin{abstract}
The experimental observation of phason fluctuations, both static and dynamic, is well established and has been interpreted within the framework of a hydrodynamical theory. Theoretically, it is still open in what temperature range a hydrodynamical description of phasons is applicable. It has been proposed in the literature that towards low temperatures the hydrodynamical regime is terminated because the form of the phason elastic energy changes from harmonic to nonanalytical. Here we report on two contributions to the field: First, for a two-dimensional binary model quasicrystal at zero temperature we have investigated the relation between atomic two-body potentials and the phason elastic energy. It turns out that there are indeed certain potentials that can induce a nonanalytic phason elastic energy. But these must have very peculiar features in their long range part rendering their existence highly unlikely. Second, one solution of the hydrodynamical equations is a phasonic excitation riding a phonon. We have studied the atomistic counterpart via a nonlinear chain with double well potentials, which resembles a dynamical fibonacci sequence. We see correlated phason flips propagating with phonon excitations and in the dynamical structure factor can separate phonon from phason dynamics.
\end{abstract}

\section{Introduction}

Tiling models of quasicrystals immediately imply the possibility of alternate vertex positions. In the cut and project algorithm vertex changes are induced by a displacement field $w\left(x^{\|}, t\right)$ of the hypercrystal along perpendicular space. The changes can be uncorrelated, and then are called phason flips, or be correlated, then denoted phason-excitations. Phason flips have been observed in triple axis neutron scattering and Mössbauer experiments [1], phason excitations by quasielastic neutron scattering [2], x-ray speckle patterns [3], and 
electron microscopy images, where they follow the path of dislocations [4]. Phason excitations carry a phason-elastic energy which depends on the phason strain tensor $\chi=\nabla w$. From tiling models it has been deduced that at low temperatures (for $d<3$ at $T=0$ ) the energy is proportional to $|\chi|$, assuming a nonanalytic form. Entropy terms modify the energy to a standard quadratic (harmonic) form with increasing temperature. This behaviour is supposed to explain the unpinning of phason excitations upon warming a quasicrystal.

In this conference contribution we investigate the phason elastic energy for $T=0$ from an atomistic point of view. We consider a two-dimensional binary model quasicrystal, the Mikulla-Roth tiling, whose atoms interact by radially symmetric pair potentials. Phason strain changes the types and frequencies of atomic neighborhoods and hence also the radial pair distribution functions. These changes can be determined by the method of atomic surfaces. Weighting the deviation of the pair distribution functions with the pair potential yields the phason elastic energy. It turns out that for a potential range up to about six atomic separations, the phason elastic energy is harmonic. For larger distances, approaching the matching rule diameter, nonanalytic terms $\propto|\chi|$ can appear. For these to become dominant, however, the potential must assume a very peculiar form (see Fig. 3) whose realization is highly improbable.

A harmonic phason elastic energy allows a hydrodynamic description of the phason excitations. At the end of the paper we comment the possibility of a phason riding a phonon. This is a solution to the hydrodynamic equations which we also find realized in our newly developed model of the "dynamical fibonacci chain (DFC)".

\section{$2 \quad$ Energy models}

Standard tilings, like the binary Mikulla-Roth tiling (MRT), are traversed by straight sequences of tile clusters ("worms"), all of which are aligned in the perfect state (in the MRT these are octagons, see Fig. 2). A uniform phason displacement causes all center vertices of selected worms to flip simultaneously. Under phason strain only parts of the worms are flipping, leaving "jags" at the sites of fracture. The number of jags per unit area is proportional to $|\chi|$. Hence for energetically stabilized tiling models also the phason elastic energy has been assumed proportional to $|\chi|$.

In two previous publications we have studied the phason (and also phonon) elastic energy. In [5] we directly applied phason and phonon strain to our binary model quasicrystal with atoms interacting by short range Lennard-Jones potentials and measured the change in total potential energy by molecular dynamics relaxation simulations. We found a harmonic energy, although with one negative elastic constant, rendering the system metastable. To improve the results, in [6] we analyzed the phason elastic constants in dependence of the two-body potentials, by counting the atomic neighborhoods as a function of phason strain. If we consider a cutoff radius $R_{c}$ for the interaction, the energy $E_{i}$ of atom $i$ depends only on the potentials $\phi$ and the atomic configuration inside the ball $B_{R_{c}, x_{i}}$ of radius $R_{c}$ around the atom location $x_{i}$. With the help of the polar calculus we could determine type and 
frequencies of atomic environments in such a ball and also the Voronoi volumes of the central atoms, and thus the energy density. The resulting expressions allowed us to modify the potentials such that absolute stability of the system was attained. Subsequent cooling Monte Carlo simulations yielded as ground state a supertile random tiling.

The cutoff radius was limited, however, to $R_{c}=1.92$ (in units of the next-neighbor bond length), but comprised already 299 symmetry inequivalent environments. When increasing $R_{c}$ this number exploded exponentially. Therefore we slightly modified the method, using the polar calculus to count not the frequency of atomic environments but of separation vectors.

\section{Phason elastic energy derived from two-body po- tentials}

The acceptance domain of the Mikulla-Roth binary tiling consists of three disconnected parts: a regular decagon for the set $A$ of large atoms, and two pentagonal stars for the sets $B_{1}$ and $B_{4}$ of small atoms. Assume an atom of type $a \in T:=\left\{A, B_{1}, B_{4}\right\}$ positioned at $\pi^{\|} r_{i}$ and an atom of type $b$ separated from it by a vector $\pi^{\|} r$, i.e. positioned at $\pi^{\|}\left(r_{i}+r\right)$. The vectors $r_{i}$ and $r$ are hyperspace vectors, $\pi^{\|}$is their projection into physical space. Then the frequency of such pairs equals the intersection $A_{a, b, \pi^{\perp}}$ of the acceptance domains of the atoms $a$ and $b$, shifted relative to each other by the projection $\pi^{\perp} r$ of $r$ into orthogonal space. A phason strain $\chi$ deforms the basis vectors of hyperspace, changing their projections into orthogonal space from $e_{i}^{\perp}$ into $e_{i}^{\perp}+\chi e_{i}^{\|}$. As the vertices of the acceptance domains are projections of hyperlattice vectors, the deformation of these domains can be calculated and also how their intersections $A_{a, b, \pi^{\perp}}(\chi)$ depend on phason strain. Given two-body potentials $\phi_{a b}$ the phason elastic energy density becomes

$$
f(\chi)=\frac{1}{V^{*}} \frac{1}{2} \sum_{|\pi||r|<R_{c}, a \in T} A_{a, b, \pi \perp_{r}}(\chi) \phi_{a b}\left(\pi^{\|} r\right)
$$

where $b$ depends on $r$. The normalizing factor $V^{*}=\sum_{a} A_{a} V_{a}$ is the sum of the Voronoi volumina $V_{a}$ of the different atom types, weighted by the volumes $A_{a}$ of their acceptance domains. As the deformation modes of orthogonal space are volume preserving, $V^{*}$ is independent on phason strain and turns out to be $25 \mathrm{~s}^{2} / \tau$, where $s$ is the volume of the fat rhomb.

Normally the intersection $A_{a, b, \pi^{\perp}}(\chi)$ is calculated for one orientation of the separation vector $r$, and we denote it for the moment by $A_{e}$. To find it for all other orientations we determine from the factor set $D_{10} / H, H$ being the fix-point group of $r$, a set of representatives $R$ which generate the orbit of $r$ under the system symmetry group $D_{10}$. The intersections of the acceptance domains of the other orientations then are calculated by the transformation

$$
g \in R: A_{g}=g A_{e}(\chi)=A_{e}\left(D\left(g^{-1}\right) \chi\right)
$$


leading to the intersection for all orientations $A=\sum_{g \in R} g A_{e}$. Now there are two types of intersections: (i) Those which have a finite value for each orientation. Under small phason strain they merely undergo an affine transformation. The group average eq. 2 cancels the linear terms and yields a quadratic (harmonic) dependence on $\chi$. (ii) For larger separations $\pi^{\|} r$ also degenerate intersections appear. These are finite for a positive sign of $\chi$, but zero for the negative sign. In the group average eq. 2, however, there is one partner, which has the opposite behaviour. The sum of the partners yields terms proportional to a sum of absolute values of components $\chi_{i j}$. In an expansion up to second order we then obtain the following form for the intersections of acceptance domains:

$$
A=a_{0}+a_{1}\left\langle\left|\chi_{01}\right|\right\rangle_{D_{10}}+a_{2} \sum_{i} \chi_{i}^{(6)^{2}}+a_{3} \sum_{i} \chi_{i}^{(8)^{2}}+a_{4}\left\langle\chi_{11}\left|\chi_{01}\right|\right\rangle_{D_{10}}
$$

where the brackets denote the group average $\langle x\rangle_{D_{10}}:=\frac{1}{20} \sum_{g \in D_{10}} g x$ and $\chi_{i}^{(6)}, \chi_{i}^{(8)}$ are the components of $\chi$ in the irreducible representations $\Gamma_{6}$ and $\Gamma_{8}$ of $D_{10}$.

Inserting eq. 3 into eq. 1 we obtain the elastic energy density

$$
f=f_{0}+\mu_{1}\left\langle\left|\chi_{01}\right|\right\rangle_{D_{10}}+\frac{1}{2} \lambda_{7} \sum_{i} \chi_{i}^{(6)^{2}}+\frac{1}{2} \lambda_{9} \sum_{i} \chi_{i}^{(8)^{2}}+\frac{1}{2} \mu_{2}\left\langle\chi_{11}\left|\chi_{01}\right|\right\rangle_{D_{10}}
$$

with elastic constants $\lambda_{7}$ and $\lambda_{9}$ for the harmonic terms. The constants $\mu_{1}$ and $\mu_{2}$ are proportional. All constants are weighted sums of potential values, e.g. $\mu_{1}=\sum_{a b, r} \beta_{a b, r} \phi_{a b}(r)$.

The coefficients $\beta_{a b, r}$ of $\mu_{1}$, which weight the linear nonanalytic term, are plotted in Fig. 1.

[ Insert figure 1 about here. ]

They show the following remarkable features: (i) They appear at large separations, more than five rhomb edges. (ii) They model exactly the matching rules. The matching rule violations in the Mikulla-Roth binary tiling are flipped octagons on a line, which have the same orientation in a perfect quasicrystal (see Fig. 2). As there are three atoms inside each octagon, there are nine inter-atomic links at six different distances which distinguish the allowed configuration from the forbidden one. The allowed separations are weighted by negative coefficients, the forbidden ones by positive coefficients. The aligned flippable octagons are spaced with three different distances, one around six rhomb edges (shown in Fig. 2), and two other roughly at 10 and 16 rhomb edges. The largest one defines the matching rule diameter (double matching rule radius).

[ Insert figure 2 about here. ]

If the potential is smooth close to these octagon spacings, then positive and negative contributions of the coefficients $\beta_{a b, r}$ cancel out and make the linear terms vanish. Only if potential oscillations alternate exactly with the $\beta$-terms, as shown in the modification of the Lennard-Jones potential in Fig. 3, then a nonanalytic form of the phason elastic energy becomes possible. Such an highly correlated fine tuning, however, is most unlikely.

[ Insert figure 3 about here. ]

Furthermore, to stabilize the quasicrystal energetically, these features of the potential have to repeat at distances around 10 and 16 rhomb edges. (If only the octagons with small distances were aligned, one would still have a supertile random tiling.) 
One therefore can state with high certainty, that if one passes from the tiling model to an atomistic point of view, a nonanlytic phason elastic energy is not occurring, rendering the entire locked state theory academic.

\section{Correlated dynamic phasons}

A harmonic phason elastic energy allows a hydrodynamic description of deformation modes in quasicrystals. The hydrodynamic equations, as derived by Lubensky et al. [7] are partial differential equations coupling the phonon and phason displacement fields $u(r, t), w(r, t)$, with the momentum, $g(r, t)$, mass, $\rho(r, t)$ and energy, $\epsilon(r, t)$, density. In the minimal model by Rochal et al. [8] - after elimination of $g$ - only $u$ and $w$ are taken into account. For longitudinal hydrodynamic excitations along the high symmetry directions of an icosahedral quasicrystal there are two types of solutions (here along the two-fold direction and treated up to order $q^{2}$ ) which always are mixed modes:

1. Two propagating modes with dispersion relation

$$
\omega(q)= \pm c q-\frac{\mathrm{i}}{2} q^{2}\left\{\eta+\frac{\Gamma_{w} K_{3}^{2}}{\Lambda}\right\}
$$

and amplitude ratio

$$
w_{0}=-\mathrm{i} \omega \frac{\Gamma_{w} K_{3}}{c^{2}} u_{0}
$$

where $c$ is the velocity of longitudinal sound, $\eta$ a momentum damping, $\Gamma_{w}$ a kinetic coefficient for phason dynamics, $\Lambda=\lambda+2 \mu$ a linear combination of the Lamé coefficients and $K_{3}$ the phason-phonon coupling constant.

2. One diffusive mode with dispersion relation

$$
\omega=-\mathrm{i} q^{2}\left(\Gamma_{w} K_{12}-\frac{\Gamma_{w} K_{3}^{2}}{\Lambda}\right)
$$

and amplitude ratio

$$
u_{0}=-\frac{K_{3}}{\Lambda} w_{0}
$$

where $K_{12}=K_{1}+K_{2}$ is the sum of the two phason-elastic constants.

Whereas the second mode is purely diffusive and phason-dominated with a small admixture of phononic deformation, the first two modes are phonon-dominated. The remarkable observation is that a phonon is accompanied by a cloud of phason flips and hence in a certain sense is inducing correlated propagating phasons. A rough estimate yields about $10^{-12}$ of the atoms flipping per picosecond.

To investigate the behaviour of phasons and their coupling to phonons we have developed the model of a "dynamical fibonacci chain". It consists of one sort of particles interacting by two-particle double well potentials with barrier height $\epsilon$ such that two equilibrium 
separations can occur: long $L$ or short $S$. Damping proportional to relative velocities can be switched on.

We have performed two different simulations [9]. In constant temperature runs where damping is neglected, the temperature dependence of the dynamic structure factor was examined. It turns out that its incoherent (self-) part possesses a Lorentzian shape at high temperatures (Fig. 4, $T_{r e d}=5.0,1.0$ and 0.5), characteristic for flip dynamics. As the temperature is lowered the width of the Lorentzian decreases, indicating smaller flip rates. In addition a further peak arises at about $\omega=8$, representing the self part for a harmonic fibonacci chain, i.e. the phonon dynamics.

[ Insert figure 4 about here. ]

In a simulation with damping but without initial temperature, we investigated the propagation of phason excitations under a forced oscillation of the left-most atom (Fig. 5). We found that the phason front propagates along the chain with pinninng and depinning and jumping over several lattice sites. Thus the phason riding a phonon has been reproduced in the discrete model of the dynamical fibonacci chain.

[ Insert figure 5 about here. ]

\section{Acknowledgments}

We thank Dr. Franz Gähler, Dipl. Phys. Dipl. Math. Michael Engel and cand. phys. Steffen Sonntag for valuable discussions.

\section{References}

[1] G. Coddens, S. Lyonnard, B. Hennion, Y. Calvayrac, Phys. Rev. B 62 (2000) 6268.

[2] M. de Boissieu, M. Boudard, B. Hennion, R. Bellissent, S. Kycia, A. Goldman, C. Janot, M. Audier, Phys. Rev. Lett. 75 (1995) 89.

[3] S. Francoual, F. Livet, M. de Boissieu, F. Yakhou, F. Bley, A. Letoublon, R. Caudron, J. Gastaldi, Phys. Rev. Lett. 91 (2003) 225501.

[4] F. Mompiou, D. Caillard, M. Feuerbacher, Phil. Mag. 84 (2004) 2777.

[5] U. Koschella, F. Gähler, J. Roth, H.-R. Trebin, J. All. Comp. 342 (2002) 287.

[6] U. Koschella, F. Gähler, J. Roth, H.-R. Trebin, J. Non-Cryst. Solids 334-335 (2004) 276.

[7] T. C. Lubensky, S. Ramaswamy, J. Toner, Phys. Rev. B 32 (1985) 7444

[8] S. B. Rochal, V. L. Lorman, Phys. Rev. B 66 (2002) 144204.

[9] A detailed report is given by M. Umezaki, H.-R. Trebin and T. Odagaki in this volume. 
Figure 2: Allowed (left) and forbidden (right) configuration of two octagons. Three of the nine distances, which contribute to the non-analytic terms, are shown.

Figure 3: Potential enforcing non-analytic behaviour and coefficients $\beta_{a b, r}$, versus $r$.

Figure 4: $\omega * S_{s}(q, \omega)$ vs $\omega$ for several temperatures. At high $T_{r e d}=k T / \epsilon(5.0,1.0$ and 0.5$)$ a single peak is observed which moves towards smaller frequencies when $T_{\text {red }}$ is lowered. It indicates a Lorentzian form of $S_{s}(q, \omega)$ with decreasing width. At $T_{r e d}=0.1$ and 0.01 the signature of a phononic resonance appears additionally at about $\omega=8$. The wavevector $q=2 * \pi /(L-S)$ is fixed.

Figure 5: Phasonic flip front versus $t$ upon periodic excitation of the left-most atom in double logarithmic representation. $k / a$ is the ratio of damping to amplitude. For zero damping the phason is running with the phonon, with increasing damping the phasons become pinned. 

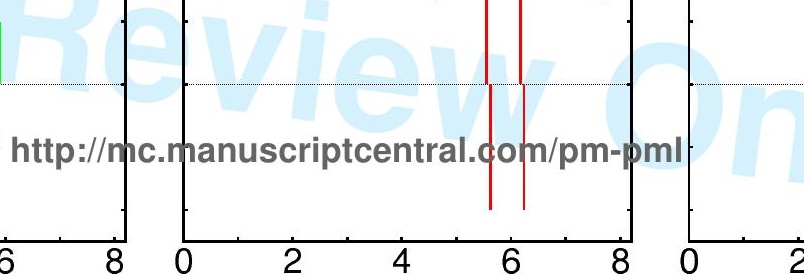


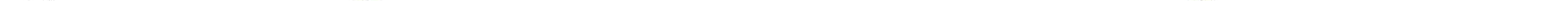


Philosophical Magazine \& Philosophical Magazine Petter\$0 of 12

1.5

1

$2^{1}$

3

045

5

6

70

8

$-095$

10

11

12

$-1 \frac{13}{4}$

15

16

170

\section{http://mc.manuscriptcentral.com/pm-pml}

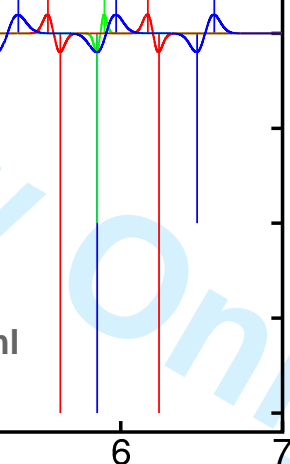




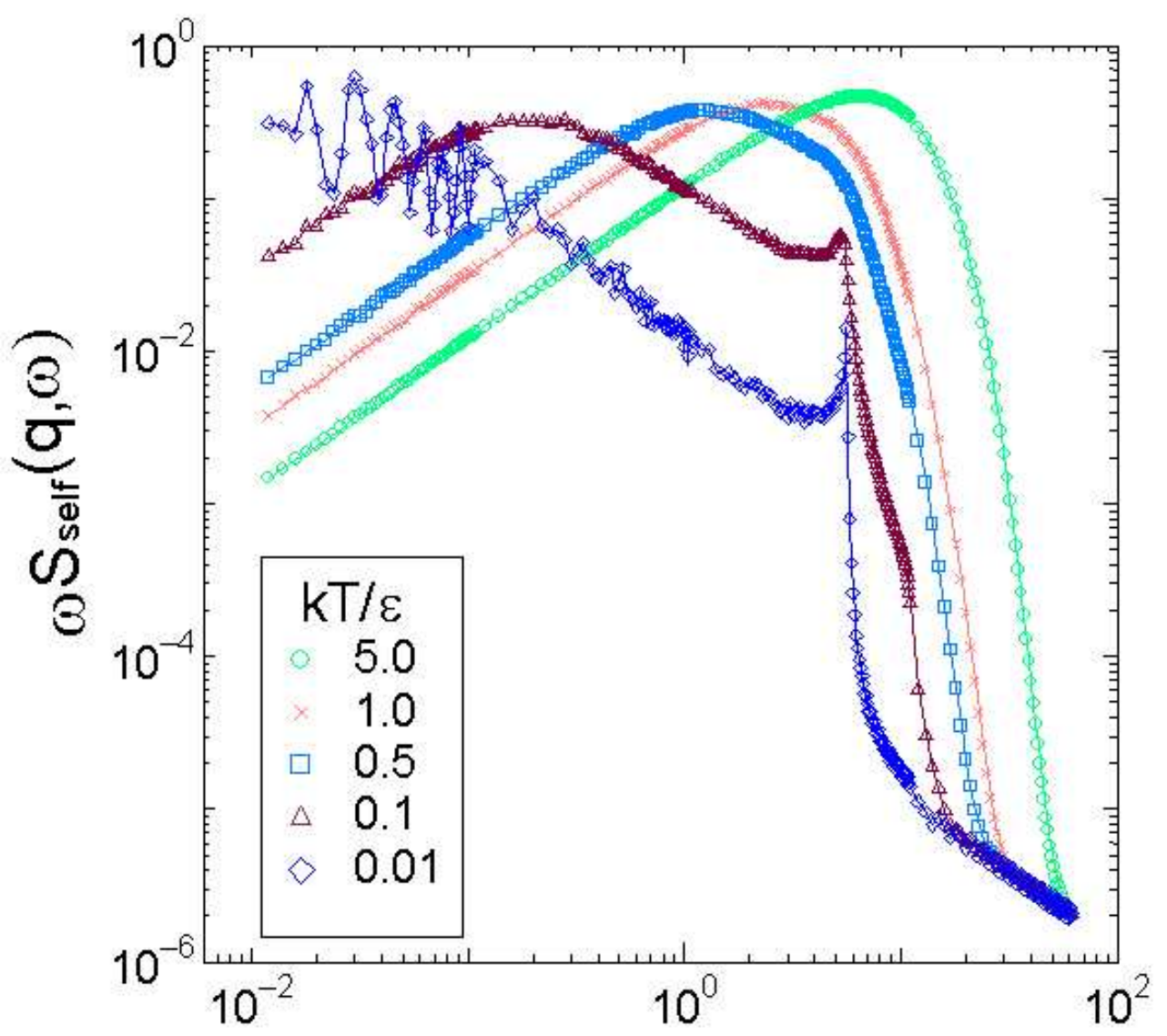

$\omega$

\$\omega*S_s(q, \omega)\$ vs \$\omega\$ for several temperatures. At high $\$ T_{-}\{$red $\}=\mathrm{kT}$ /epsilon $\$(5.0,1.0$ and 0.5$)$ a single peak is observed which moves towards smaller frequencies when $\$ T$ _red $\$$ is lowered. It indicates a Lorentzian form of $\$ S \_s(q$, lomega $) \$$ with decreasing width. At $\$ T \_\{\text {red }\}=0.1 \$$ and 0.01 the signature of a phononic resonance appears additionally at about $\$ \backslash$ mega $=8 \$$. The wavevector $\$ q=2 * \backslash p i /(L-S) \$$ is fixed. $211 \times 198 \mathrm{~mm}(72 \times 72 \mathrm{DPI})$ 


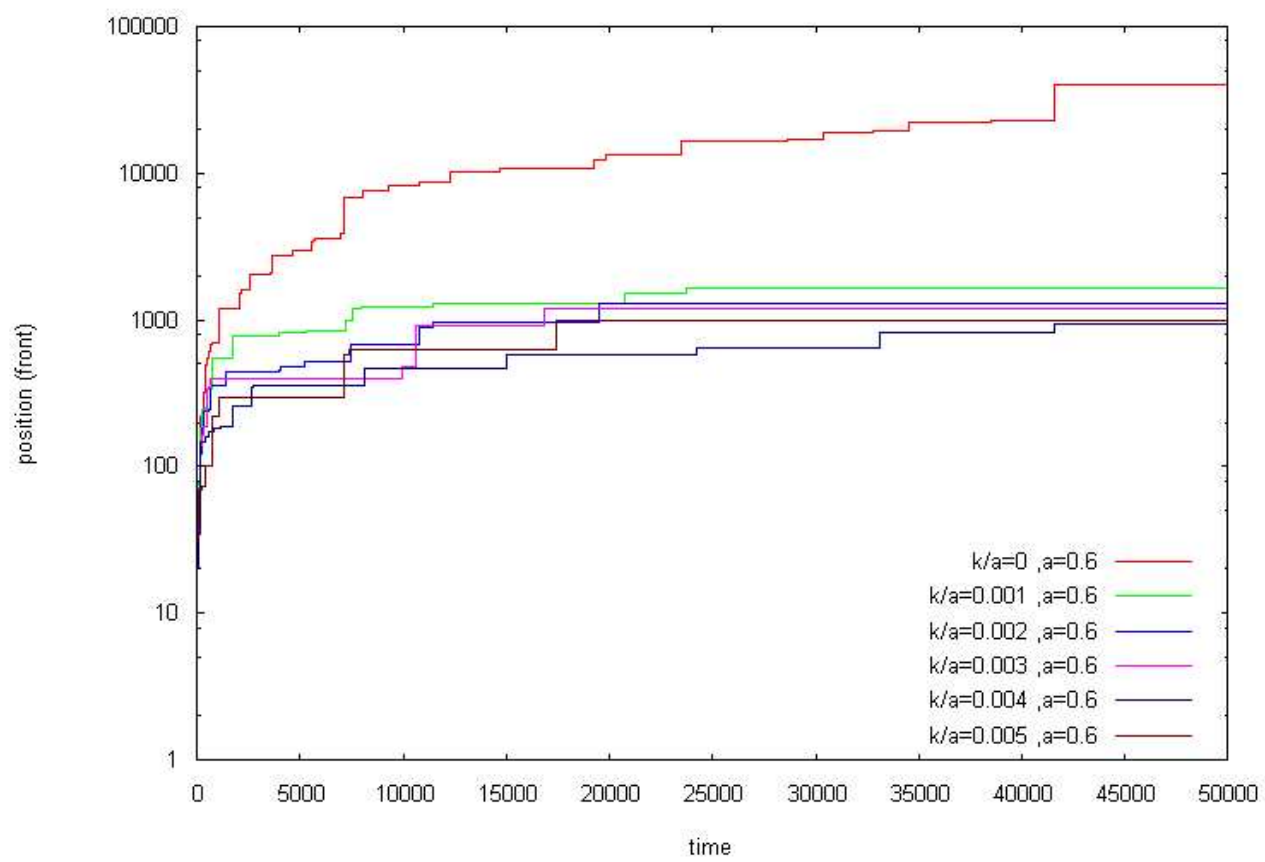

Phasonic flip front versus $\$$ t\$ upon periodic excitation of the left-most atom in double logarithmic representation. $\$ \mathrm{k} / \mathrm{a} \$$ is the ratio of damping to amplitude. For zero damping the phason is running with the phonon, with increasing damping the phasons become pinned. $268 \times 179 \mathrm{~mm}(72 \times 72 \mathrm{DPI})$ 